\title{
Robust control of identified models with mixed parametric and non-parametric uncertainties (revised version)
}

\author{
Wolfgang Reinelt, Lennart Ljung \\ Division of Automatic Control \\ Department of Electrical Engineering \\ Linköpings universitet, SE-581 83 Linköping, Sweden \\ WWW: http://www. control.isy.liu.se \\ Email: wolle@isy.liu.se, ljung@isy.liu.se
}

May 2001

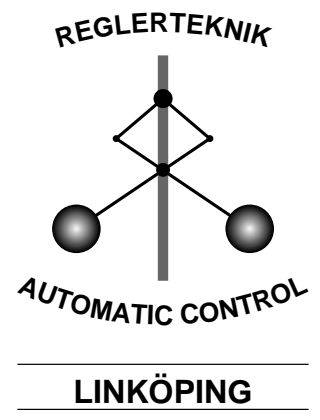

Report No.: LiTH-ISY-R-2352

Submitted to ECC 2001, Porto, Portugal

Technical reports from the Automatic Control group in Linköping are available by anonymous ftp at the address ftp.control.isy.liu.se. This report is contained in the file 2352.pdf. 


\title{
ROBUST CONTROL OF IDENTIFIED MODELS WITH MIXED PARAMETRIC AND NON-PARAMETRIC UNCERTAINTIES
}

\author{
Wolfgang Reinelt and Lennart Ljung \\ Dept of Electrical Engineering, Linköping University, 58183 Linköping, Sweden. \\ Fax: +46 13 282622. E-mail: wolle,ljung@isy.liu.se
}

Keywords: Robust Stability and Performance, Rank One Uncertainties, Model Error Modeling, Identification for Control.

\begin{abstract}
A framework for identification oriented robust controller design is developed. The model is identified from open-loop i/odata and contains parametric uncertainties as well as an additive and norm-bounded error. The set of all robustly stabilising controllers, that additionally guarantee robust performance is characterised by a system of second order cones, which can be efficiently solved by interior point algorithms.
\end{abstract}

\section{Introduction and Motivation}

Considerable current interest in System Identification is dedicated to Identification for Control, e.g. [6] and the references therein. The gap to bridge between identification and control is the model uncertainty, which will be addressed by a robust controller. Here, we are facing a fundamental robustnessperformance tradeoff, as a too "large" model uncertainty will lead to a conservative (or even impossible) controller design, while a too "small" model set may not include the real system. Validating a given controller for stability and performance for an uncertain identified model is well understood [3]. Still, there is a need for more design techniques, directly based on identification techniques delivering a nominal model and model uncertainty.

Mainstream works on robust control like $\mathcal{H}_{\infty}$ techniques mainly focus on unstructured uncertainties and are therefore not necessarily well suited for parametric uncertainties and additive, norm bounded errors from identification, cf. [5, 11].

In [17], the authors consider unstructured uncertainties described by confidence bounds and present a mixed $\mathcal{H}_{2} / \mathcal{H}_{\infty}$ framework. The main idea in this approach is to have a "nominal" controller at hand, which this will be changed in order to guarantee a-priori specified performance bounds.

[7] employs the concept of Integral Quadratic Constraints (IQCs) to handle mixed uncertainties. This approach, however, discusses the analysis of robust stability properties, given a description via IQCs.

Another approach is to study so-called interval plants (i.e. the coefficients of the transfer function live in an interval) and apply Kharitonov type of results for stability analysis, see [1]. Works dealing with actual design techniques for these types of uncertainties are, however, limited to low order controller with a fixed structure, see [10] for instance. For a treatment of mixed uncertainties in this framework see [4]. A related work is [14], where interval arithmetic is applied to analyse model sets that depend nonlinearly on some uncertain parameters.

However, a necessary and sufficient result on robust stability for rank one uncertainties is given in [16] and we will exploit this result to arrive at a design procedure, guaranteeing robust stabilisation of the model, containing real parametric uncertainties as well an additive norm-bounded error. Moreover, robust sensitivity can be prescribed as usual in $\mathcal{H}_{\infty}$ control and additionally, the sensitivity of the nominal control system can be shaped within certain (sharper) bounds. The problem is formulated as a second order cone programming problem, which enables an efficient numerical solution.

An approach, based on the same stability result can be found in [2]. There, the authors consider the maximisation of the stability margin for a class of restricted order controllers.

Paper Outline: Sec. 2 summarises short the result of a identification procedure, while Sec. 3 then discusses controller design ensuring robust stability and performance. We illustrate our approach with an example in Sec. 4 and give some closing remarks in Sec. 5.

\section{What the identification procedure delivers}

We consider single-input single-output models, that are linearly parameterised, i.e. we assume the structure $G=B \cdot \theta$ for the i/o behaviour, where $B$ denotes the vector of chosen basis functions, and $\theta$ the parameter vector, to be estimated from data. Doing so, we end up with a nominal model $G_{n o m}=B \cdot \theta_{\text {nom }}$ and the model set:

$$
\begin{aligned}
\mathcal{U}_{p}:=\{G(\theta) & ; G(\theta):=B \cdot \theta, \\
& \left.\left(\theta-\theta_{\text {nom }}\right)^{T} E^{-1}\left(\theta-\theta_{\text {nom }}\right) \leq \rho\right\}
\end{aligned}
$$

Applying Set Membership techniques, assuming an unknownbut-bounded noise, the positive definite matrix $E$ in (1) refers to the ellipsoid, calculated when using an ellipsoidal overbounding algorithm [15] and $\rho=1$. Using Least Squares techniques [12], $E$ is the covariance matrix of the parameter and $\rho$ is linked to the probability level of estimation.

The dynamics of the model set $\mathcal{U}_{p}$ depend of those of the basis $B$, which hinges on a-priori knowledge. Even if the main dynamics are picked up here, under-modelling, nonlinearities 


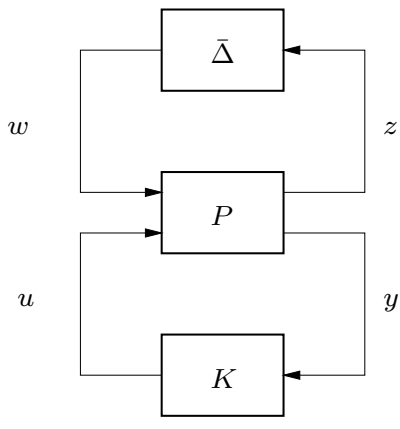

Figure 1: Generalised plant $P$ with uncertainty $\bar{\Delta}$ and controller $K$.

or time varying drifts may appear. Therefore, one usually estimates an un-falsified, additive, norm bounded model error for the nominal model $[11,5]$. The result is then

$$
\mathcal{U}_{n p}:=\left\{G_{\Delta} ; G_{\Delta}:=G_{n o m}+W_{\Delta} \Delta,\|\Delta\|_{\infty} \leq 1\right\}
$$

Clearly, both model uncertainties discussed so far are of different nature: the model set $\mathcal{U}_{p}$ is represented by a real parametric uncertainty, bounded in 2-norm, while the model set $\mathcal{U}_{n p}$ represents a non-parametric uncertainty, bounded in $\mathcal{H}_{\infty}$ norm. Obviously, it is useful to combine both types of uncertainty set to cope with uncertainties in the parameters of the nominal model as well as with unmodeled dynamics, which leads to

$$
\begin{aligned}
\mathcal{U} & :=\left\{G ; G=B \cdot \theta+W_{\Delta} \Delta\right. \\
& \left.\left(\theta-\theta_{\text {nom }}\right)^{T} E^{-1}\left(\theta-\theta_{\text {nom }}\right) \leq \rho,\|\Delta\|_{\infty} \leq 1\right\}
\end{aligned}
$$

\section{Controller Design}

\subsection{Robust Stability and Performance}

The model set (3) fits to standard uncertainty descriptions (cf. Fig. 1), when resorting the two contributions of uncertainty to $\bar{\Delta}$. Therefore it is possible to use control schemes like mixed sensitivity $\mathcal{H}_{\infty}$ control, exploiting the uncertainty band in the frequency domain. But here, we are in a much more specific situation: the uncertainty $\bar{\Delta}$ consists of two contributions. Firstly, the real uncertainty vector $\delta$, bounded by an ellipsoid and can hence be described as $\|\delta\|_{2} \leq 1$, denoting $\delta=\frac{1}{\sqrt{\rho}} T\left(\theta-\theta_{\text {nom }}\right), T \cdot T=E^{-1}$. Secondly, the nonparametric uncertainty bounded in operator norm. Thus, the uncertainty of model set (3) is a rank one uncertainty $\left(\delta^{T}, \Delta\right)$ with a generalised plant $P$, that is known from the identification procedure. Additionally to robust stability, we ask the controller to guarantee a certain level of performance for the identified model set (3). In particular we demand an upper bound $W_{S}$ for the sensitivity function $S$ : $\left\|W_{S}^{-1} \cdot S\left(\delta^{T}, \Delta\right)\right\|_{\infty} \leq 1, \forall\|\delta\|_{2} \leq$ $1,\|\Delta\|_{\infty} \leq 1$, which is equivalent to robust stability by adding a dummy uncertainty $\Delta_{S}$ to the uncertainty vector and then applying the small gain theorem [9, Sec.3.4]. We then arrive at the following situation:

$$
\begin{aligned}
\left(\begin{array}{c}
z \\
y
\end{array}\right) & \left.=\left(\begin{array}{c}
0 \\
0 \\
W_{S}^{-1} \\
1
\end{array}\right)\left(\begin{array}{c}
\sqrt{\rho} T^{-T} B^{T} \\
W_{\Delta} \\
W_{S}^{-1} G_{n o m} \\
G_{n o m}
\end{array}\right)\right)\left(\begin{array}{c}
w \\
u
\end{array}\right) \\
& =P\left(\begin{array}{c}
w \\
u
\end{array}\right) \\
w & =\bar{\Delta} z=\left(\delta^{T}, \Delta, \Delta_{S}\right) z \\
& \|\delta\|_{2} \leq 1,\|\Delta\|_{\infty} \leq 1,\left\|\Delta_{S}\right\|_{\infty} \leq 1
\end{aligned}
$$

We now aim at using the convex parameterisation of all stabilising controllers, see [16]. Suppose, we have a Youla parameterisation of the nominal i/o behaviour from $w$ to $z: T_{1}+T_{2} Q$, where $Q$ is the Youla parameter. Assume moreover a decomposition of $\left(T_{1}, T_{2}\right)$ corresponding to the uncertain vector $\left(\delta^{T}, \Delta, \Delta_{S}\right)^{T}:\left(T_{1}, T_{2}\right)=\left(\begin{array}{ll}T_{1}^{\delta} & T_{2}^{\delta} \\ T_{1}^{\Delta} & T_{2}^{\Delta} \\ T_{1}^{\Delta_{S}} & T_{2}^{\Delta_{S}}\end{array}\right)$. Robust stability for the feedback loop is now to demand:

$$
\left[1-\left(\delta^{T}, \Delta, \Delta_{S}\right)\left(T_{1}+T_{2} Q\right)\right]^{-1} \in \mathcal{H}_{\infty}, \forall \delta, \Delta, \Delta_{S},
$$

but a computationally more attractive formulation of this robust stability problem is:

Theorem 1 ([16]) Under the assumptions described so far, (6) is equivalent to the existence of two stable functions $\alpha, \beta$ with $Q=\beta \alpha^{-1}$ fulfilling

$$
\begin{aligned}
& \left\|\operatorname{Re}\left[T_{1}^{\delta} \alpha+T_{2}^{\delta} \beta\right](i \omega)\right\|^{d}+\left\|\left(T_{1}^{\Delta} \alpha+T_{2}^{\Delta} \beta\right)(i \omega)\right\|_{2}+ \\
& \left\|\left(T_{1}^{\Delta_{S}} \alpha+T_{2}^{\Delta_{S}} \beta\right)(i \omega)\right\|_{2}<\operatorname{Re} \alpha(i \omega)
\end{aligned}
$$

for all frequencies $\omega$, where $\|\cdot\|^{d}$ is denotes the dual norm to $\|\cdot\|$.

Remark 1 The original formulation [16, Cor.2] is stated for one dynamic uncertainty, i.e. $\Delta_{S}=0$ in the above setup, which is straightforward to extend.

The remaining problem is to find the two functions $\alpha, \beta$ in the now convex setting of (7). In [8], this problem is solved for a parametric uncertainty (i.e. $T_{1}^{\Delta}, T_{2}^{\Delta}, T_{1}^{\Delta_{S}}, T_{2}^{\Delta_{S}}=0$ ) bounded in 1-norm and $\infty$-norm respectively using an FIR basis for $\alpha, \beta$ and the computational problem is recasted to a Linear Programming one. Several reasons encourage us to abandon this existing solution. Apart from the missing additive error $\Delta$ and the performance specification, in our case, the uncertainty is bounded in the 2-norm, so that $\|\cdot\|^{d}=\|\cdot\|=\|\cdot\|_{2}$. Moreover, we will assume a more general parameterization in terms of bases $B_{\alpha}, B_{\beta}$ of dimension $N_{\alpha}$ and $N_{\beta}$ respectively, i.e. $\alpha=B_{\alpha} \theta_{\alpha}, \beta=B_{\beta} \theta_{\beta}, \theta_{\alpha} \in \mathbb{R}^{N_{\alpha}}, \theta_{\beta} \in \mathbf{R}^{N_{\beta}}$. Obviously, (7) is then (assuming infinite basis expansion: $N_{\alpha}, N_{\beta} \rightarrow \infty$ ) equivalent to

$$
\left\|\mathcal{R}_{s} \theta\right\|_{2}+\left\|\mathcal{R}_{u s} \theta\right\|_{2}+\left\|\mathcal{R}_{P} \theta\right\|_{2}<\mathcal{R}_{m}^{T} \theta, \quad \forall \omega
$$


denoting $\theta^{T}=\left(\theta_{\alpha}^{T}, \theta_{\beta}^{T}\right)$ and (the dependence on frequency $\omega$ being suppressed):

$$
\mathcal{R}_{s}=\left(R e T_{1}^{\delta} B_{\alpha}, \operatorname{Re} T_{2}^{\delta} B_{\beta}\right), \mathcal{R}_{u s}=\left(\begin{array}{cc}
\operatorname{Re} T_{1}^{\Delta} B_{\alpha} & \operatorname{Re} T_{2}^{\Delta} B_{\beta} \\
\operatorname{Im} T_{1}^{\Delta} B_{\alpha} & \operatorname{Im} T_{2}^{\Delta} B_{\beta}
\end{array}\right.
$$

$$
\mathcal{R}_{P}=\left(\begin{array}{cc}
\operatorname{Re} T_{1}^{\Delta_{S}} B_{\alpha} & \operatorname{Re} T_{2}^{\Delta^{S}} B_{\beta} \\
\operatorname{Im} T_{1}^{\Delta_{S}} B_{\alpha} & \operatorname{Im} T_{2}^{\Delta^{S}} B_{\beta}
\end{array}\right), \mathcal{R}_{m}=\operatorname{Re} B_{\alpha}^{T}
$$

Eqn. (8) is equivalent to the following second order cone, when introducing additional dummy-variables, i.e. $\tilde{\theta}=$ $\left(\theta^{T}, \zeta_{1}, \zeta_{2}, \zeta_{3}\right)^{T}$ :

$$
\begin{aligned}
0 & <\tilde{\mathcal{R}}_{m}^{T} \tilde{\theta} \\
\left\|\tilde{\mathcal{R}}_{s} \tilde{\theta}\right\|_{2} & \leq(\underline{0}, 1,0,0) \tilde{\theta} \\
\left\|\tilde{\mathcal{R}}_{u s} \tilde{\theta}\right\|_{2} & \leq(\underline{0}, 0,1,0) \tilde{\theta} \\
\left\|\tilde{\mathcal{R}}_{P} \tilde{\theta}\right\|_{2} & \leq(\underline{0}, 0,0,1) \tilde{\theta}
\end{aligned}
$$

Here, $\underline{0}$ denotes the row vector with $N_{\alpha}+N_{\beta}$ zero entries and obvious blocks have been added. This problem can be solved quite efficiently with standard software as SOCP, see [13].

\subsection{Imposing additional (sharper) nominal performance}

In the particular situation that identification is performed using a set of stable basis functions it is straightforward to impose additional demands on the sensitivity function of the nominal control system, which is then $S=1-G_{n o m} Q$. Assume therefore non-negative lower and upper bounds $S_{l o}(i \omega), S_{h i}(i \omega)$ for the sensitivity, which is equivalent to

$$
\begin{aligned}
\left|S_{l o}(i \omega) \alpha(i \omega)\right| & \leq\left|\alpha(i \omega)-G_{n o m}(i \omega) \beta(i \omega)\right| \\
& \leq\left|S_{h i}(i \omega) \alpha(i \omega)\right|, \quad \forall \omega \\
\Leftrightarrow\left\|S_{l o}(i \omega) \mathcal{S}_{\alpha}(i \omega) \theta_{\alpha}\right\|_{2} & \leq\left\|\mathcal{S}(i \omega)\left(\theta_{\alpha}^{T}, \theta_{\beta}^{T}\right)^{T}\right\|_{2} \\
& \leq\left\|S_{h i}(i \omega) \mathcal{S}_{\alpha}(i \omega) \theta_{\alpha}\right\|_{2}, \quad \forall \omega,
\end{aligned}
$$

when using the above basis functions and denoting (suppressing the dependence on $\omega$ ):

$$
\mathcal{S}_{\alpha}=\left(\begin{array}{c}
\operatorname{Re} B_{\alpha} \\
\operatorname{Im} B_{\alpha}
\end{array}\right) \quad, \quad \mathcal{S}=\left(\begin{array}{cc}
\operatorname{Re} B_{\alpha} & -\operatorname{Re} G_{n o m} B_{\beta} \\
\operatorname{Im} B_{\alpha} & -\operatorname{Im} G_{n o m} B_{\beta}
\end{array}\right) .
$$

For consistency with the variable $\tilde{\theta}=\left(\theta_{\alpha}^{T}, \theta_{\beta}^{T}, \zeta_{1}, \zeta_{2}\right)^{T}$ from above, we add obvious zero blocks to matrices $\mathcal{S}_{\alpha}, \mathcal{S}$ and obtain an equivalent formulation of (14): $\left\|S_{l o} \tilde{\mathcal{S}}_{\alpha} \tilde{\theta}\right\|_{2} \leq\|\tilde{\mathcal{S}} \tilde{\theta}\|_{2} \leq$ $\left\|S_{h i} \tilde{\mathcal{S}}_{\alpha} \tilde{\theta}\right\|_{2}$. For an easier argumentation, we break this inequality into four inequalities:

$$
\begin{aligned}
\left\|S_{l o} \tilde{\mathcal{S}}_{\alpha} \tilde{\theta}\right\|_{2} & \leq \gamma_{l o} \leq\|\tilde{\mathcal{S}} \tilde{\theta}\|_{2}, \quad \forall \omega, \\
\|\tilde{\mathcal{S}} \tilde{\theta}\|_{2} & \leq \gamma_{h i} \leq\left\|S_{h i} \tilde{\mathcal{S}}_{\alpha} \tilde{\theta}\right\|_{2}, \quad \forall \omega .
\end{aligned}
$$

For fixed values of $\gamma_{l o}, \gamma_{h i}$, the left inequalities in $(15,16)$ describe convex sets, whereas the right inequalities in $(15,16)$ do not. Inequality (14), however, can be scaled in $\left(\theta_{\alpha}^{T}, \theta_{\beta}^{T}\right)^{T}$, so that it is equivalent to demand that one of the components of the vectors $\tilde{\mathcal{S}} \tilde{\theta}$ and $S_{h i} \tilde{\mathcal{S}}_{\alpha} \tilde{\theta}$ has to have at least the demanded size $\gamma_{l o}$ and $\gamma_{h i}$ respectively. Denoting $e_{l}$ the $l-t h$ unit vector, all this leads to

$$
\begin{aligned}
\left\|S_{l o} \tilde{\mathcal{S}}_{\alpha} \tilde{\theta}\right\|_{2} & \leq \tilde{\mathcal{S}} \cdot e_{j} \cdot \tilde{\theta} \\
\|\tilde{\mathcal{S}} \tilde{\theta}\|_{2} & \leq S_{h i} \tilde{\mathcal{S}}_{\alpha} \cdot e_{k} \cdot \tilde{\theta} .
\end{aligned}
$$

\subsection{Controller design as second order cone feasibility problem}

Imposing robust stability and performance, all feasible controllers (represented by $\theta_{\alpha}, \theta_{\beta}$ ) are given by restrictions $(9,10,11,12,17,18)$, making up a second order cone:

$$
\begin{aligned}
0 & <\tilde{\mathcal{R}}_{m}^{T} \tilde{\theta} \\
\left\|\tilde{\mathcal{R}}_{s} \tilde{\theta}\right\|_{2} & \leq(\underline{0}, 1,0,0) \tilde{\theta} \\
\left\|\tilde{\mathcal{R}}_{u s} \tilde{\theta}\right\|_{2} & \leq(\underline{0}, 0,1,0) \tilde{\theta} \\
\left\|\tilde{\mathcal{R}}_{P} \tilde{\theta}\right\|_{2} & \leq(\underline{0}, 0,0,1) \tilde{\theta} \\
\left\|S_{l o} \tilde{\mathcal{S}}_{\alpha} \tilde{\theta}\right\|_{2} & \leq \tilde{\mathcal{S}} \cdot e_{j} \cdot \tilde{\theta} \\
\|\tilde{\mathcal{S}} \tilde{\theta}\|_{2} & \leq S_{h i} \tilde{\mathcal{S}}_{\alpha} \cdot e_{k} \cdot \tilde{\theta}
\end{aligned}
$$

For the numerical solution of the problem using second order cones, powerful interior point algorithms can be employed, see [13]. However, the above system can be formulated (or solved) in terms of Linear Matrix Inequalities (LMIs) as well. Note that the numerical solution in terms of second order cones is much more efficient than using LMIs.

Special cases of the design problem arise when no parametric uncertainty or no explicit model error model is present: $\tilde{\mathcal{R}}_{s}=0$ and $\tilde{\mathcal{R}}_{u s}=0$ in $(20,21)$ respectively. $\mathcal{R}_{P}=0$ in (22) imposes no further demands on robust sensitivity. Moreover $S_{l o}=0$ and $1 / S_{h i}=0$ in $(23,24)$ respectively indicate absence of lower and upper bounds for nominal sensitivity.

\subsection{Discussion}

Choice of the basis $B_{\alpha}$. Eqn. (7) implies strict positive realness of the function $\alpha$, which is easy seen from letting $\rho=0$. Suppose, we have a state space representation of $B_{\alpha} \stackrel{z}{=}(A, B, C, D)$. Then, the discrete time version of the positive real lemma [9, Prob.3.25] (using the bilinear transformation $z=(1+s) /(1-s)$ to derive it from the continuous time version, cf. [9, Prob.4.18]) states, that a necessary condition for this is $\left[D-C(I+A)^{-1} B\right]+[(D-C(I+$ $\left.A)^{-1} B\right]^{T}>0$. In the SISO case, this will be equivalent with $D-C(I+A)^{-1} B>0$. Now, suppose a first order Laguerre basis with pole at $-1<a<1: B_{\alpha} \stackrel{z}{=}\left(a, 1, \sqrt{\left(1-a^{2}\right)}, 0\right)$, then $\alpha \stackrel{z}{=}\left(a, 1, \sqrt{\left(1-a^{2}\right)} \theta_{\alpha}, 0\right)$ for some scalar parameter $\theta_{\alpha}$. The 


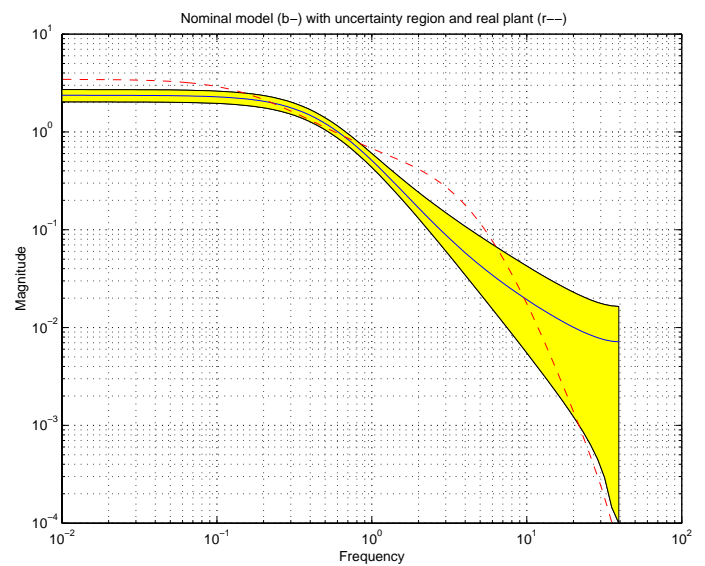

Figure 2: Nominal model (solid), uncertainty region arising from parametric uncertainty (shaded) and real plant (dashed).

above necessary condition for strict positive realness is then $\theta_{\alpha} \sqrt{\frac{1-a}{1+a}}<0$, which implies $\theta_{\alpha}<0$. Additionally, the gap between lhs and rhs achieves its maximum, when $a \searrow-1$, which hints to the fact that the choice of this Laguerre pole for $\alpha(z)$ makes inequality (7) for robust stability least restrictive.

Multivariable Systems. We restricted our investigations to SISO systems. In principle this is not necessary, as we only have to demand that the signal $w$ is scalar, which has no direct implications on the number of in- or outputs of the plant $G$. However, allowing MIMO systems, (1) would imply that we use one multivariable basis and one uncertainty vector $\delta$, rather than different bases for all components. Moreover it can be easily seen, that allowing a non-parametric additive error $\Delta$ will restrict us to MISO plants, and encountering for robust sensitivity will restrict us to SISO plants.

\section{Simulation Example}

Identification. We use the experimental environment as in [5]. 4000 data points are collected and a second order nominal model, based on a Laguerre expansion (with pole at $z=0.96$ ) is identified. Assuming an unknown but bounded noise of size $\delta_{n}=4.9$ in an ellipsoidal over-bounding algorithm leads to the nominal model $G_{n o m}(z)=0.016(z-0.76) /(z-0.96)^{2}$ and the coefficients in the Laguerre expansion are bounded in an ellipsoid of volume 0.06 .

In order to cope with unmodeled dynamics, we identify an additive error. We parameterise the set of model errors $\Delta$ by a Laguerre basis of order 25 and following the strategy described in [5], a pole location of $z=0.95$ produces a minimal, nonfalsifying error bound of $\delta_{e}=1.3$. To calculate the generalised plant (4), we need to describe the maximum amplitude of the model error by a weighting function $W_{\Delta}$. As the complexity of this weighting function will affect the complexity of the controller, we choose a rather rough and low $(9 t h)$ order weight. The result of the identification is reported in Figs. 2 and 3.
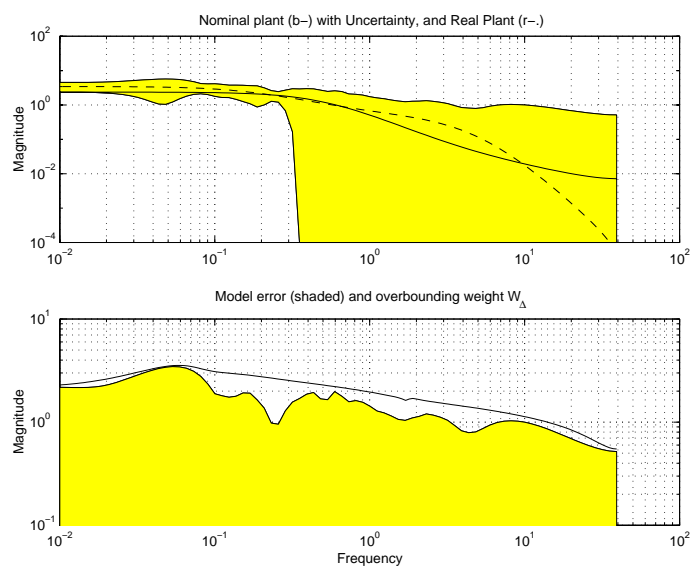

Figure 3: Upper plot: Nominal model (solid) along with uncertainty region, arising from the additive model error (shaded) and real plant (dashed). Lower plot: Nominal error (shaded) and over-bounding weight $W_{\Delta}$ (solid).

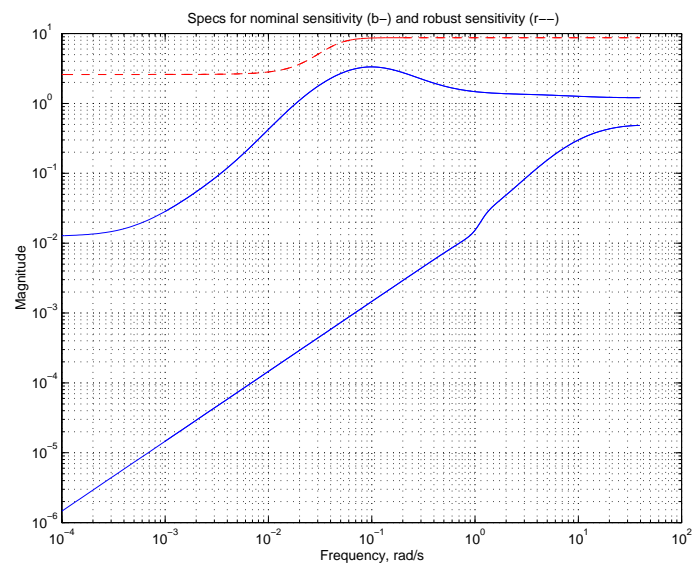

Figure 4: Upper and lower bound for the nominal sensitivity (solid) and upper bound $W_{S}$ for robust sensitivity (dashed). 

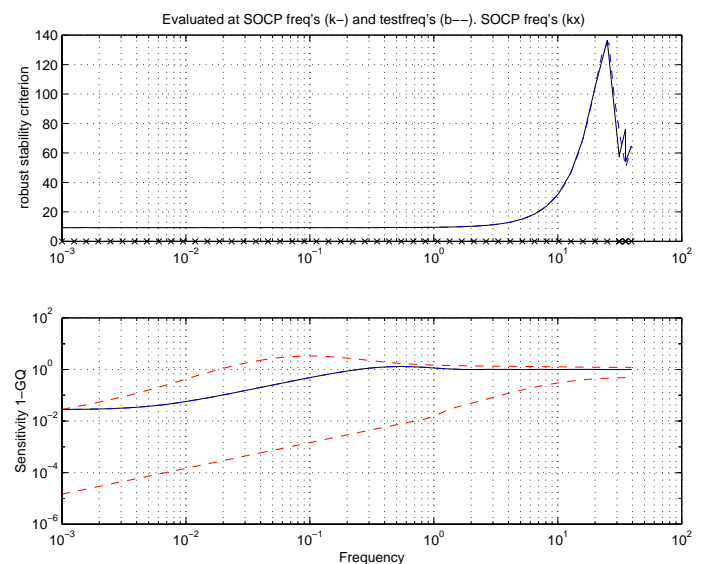

Figure 5: Full order robustly stabilising controller with robust sensitivity. Upper plot: Criterion function for robust stability (rhs of (19)), evaluated at a set of test frequencies (crosses). Lower plot: nominal sensitivity function (solid) and desired upper and lower nominal bounds (dashed).

Specifications. Additionally to robust stability for all models in th identified model set, we demand the nominal sensitivity function to stay between a lower and an upper bound and the robust sensitivity function to stay below a certain bound, see Fig. 4.

Controller design. First, we will investigate the recommendation for the choice of the basis functions $\alpha, \beta$ for the Youla parameter. We restrict the length of the Laguerre bases to $N_{\alpha}=N_{\beta}=6$. Choosing the pole of $\alpha$ to $p_{\alpha}>-0.7$ (and arbitrary $p_{\beta}$ ) results in an empty feasible set of solutions (testing a reasonable grid). In contrast, the choice $p_{\alpha}=-0.8, p_{\beta}=0.6$ leads to a robustly stabilising controller of order 21 , fulfilling all requirements posed on nominal and robust sensitivity. This supports the remark on pole choice for $B_{\alpha}$ close to -1 given in Sec. 3. The result of the controller design is reported in Fig. 5, whereas the magnitude of this controller is reported in Fig. 6.

Now, we investigate the possibility of controller reduction. We reduce the above controller to a second and first order controller respectively (Schur method model reduction). The magnitudes are reported in Fig. 6. How about robust stability and sensitivity of these controllers ${ }^{1}$ ? Our framework allows us to test a given controller for robustness and performance. Starting off with the controller, we re-calculate the corresponding Youla parameter and its coefficients for a representation via basis functions as in Theorem 1. Now, (8) enables us to check, whether this coefficient is a feasible solution or not: For each frequency, the contributions corresponding to parametric error $\left\|\mathcal{R}_{s} \theta\right\|_{2}$, additive error $\left\|\mathcal{R}_{u s} \theta\right\|_{2}$ and robust performance $\left\|\mathcal{R}_{P} \theta\right\|_{2}$ must, in sum, be less than $\mathcal{R}_{m}^{T} \theta$ (note, that this is necessary and sufficient condition).

We apply this machinery to the second order controller. As

${ }^{1}$ To check nominal sensitivity in this context is trivial and therefore not discussed here.

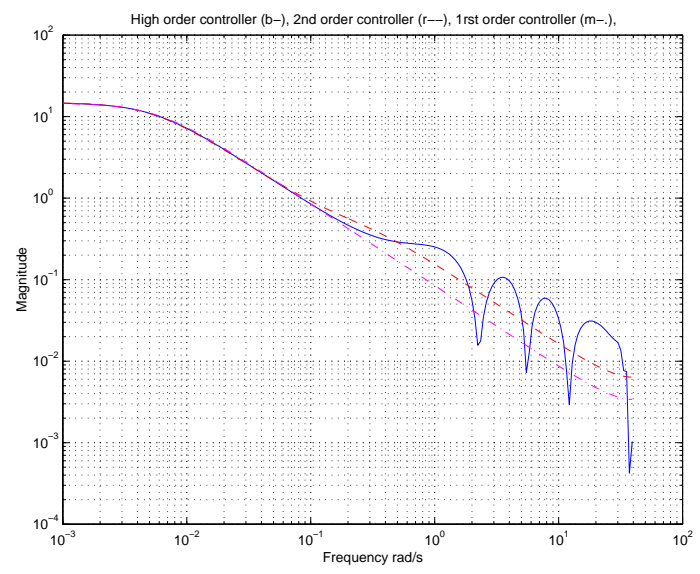

Figure 6: Magnitude of full order controller (solid), second order controller (dashed) and first order controller (dash-dotted).

(8) is fulfilled for all frequencies, we can apply this controller, guaranteeing robust stability and sensitivity. Doing the same with the first order controller, we obtain a negative answer. According to Figs. 7,8, (8) does not hold in the mid and high frequency range (at frequencies marks with stars). However, a closer look on the single contributions (Fig. 8, lower plot) enables us to sort out the constraint causing this failure ("at each frequency, all non-solid lines must add up to something less that the solid line"): Indeed, we see the the contribution of $\left\|\mathcal{R}_{P} \theta\right\|_{2}$ is too large, meaning that this first order controller ensures robust stability, but not robust sensitivity. (This does not mean that there is no first order controller that would do the job.)

\section{Conclusions}

We presented a straightforward framework for identification oriented robust controller design. The model uncertainty is described by parametric, as well as additive norm-bounded uncertainty. The set of all robustly stabilising controllers, that additionally guarantee robust performance, is characterised by a system of second order cones, which can be efficiently solved using interior point algorithms. Moreover, an already existing controller (for instance a controller obtained by model reduction) can be tested for robust stability and performance in this framework.

\section{Acknowledgement}

Financial support by the European Commission through the program Training and Mobility of Researchers - Research Networks and through the project System Identification (FMRX CT98 0206) and discussions with participants in the European Research Network System Identification, A. Ghulchak, A. Helmersson and J. Löfberg are gratefully acknowledged. 

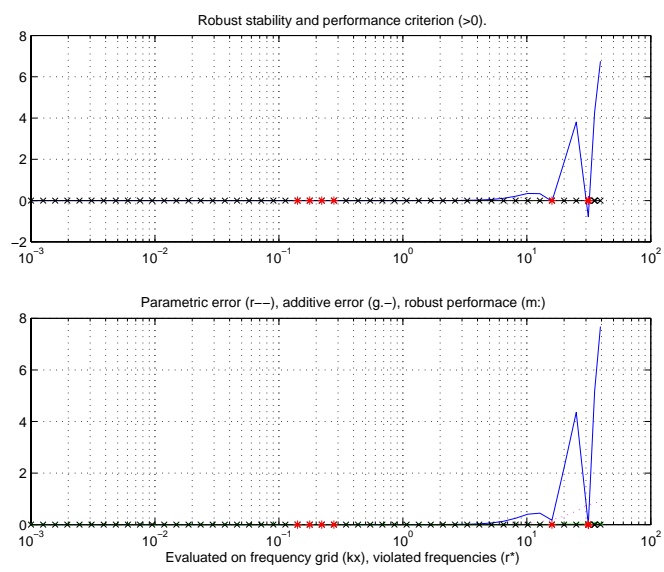

Figure 7: Analysis of first order controller (full frequency range). Upper plots: Criterion function for robust stability (rhs of (19)), evaluated at a set of test frequencies (crosses). Lower plots: Single contributions in (8): $\mathcal{R}_{m}^{T} \theta$ (solid), Parametric error $\left\|\mathcal{R}_{s} \theta\right\|_{2}$ (dashed), additive error $\left\|\mathcal{R}_{u s} \theta\right\|_{2}$ (dash-dotted), robust performance $\left\|\mathcal{R}_{P} \theta\right\|_{2}$ (dotted)
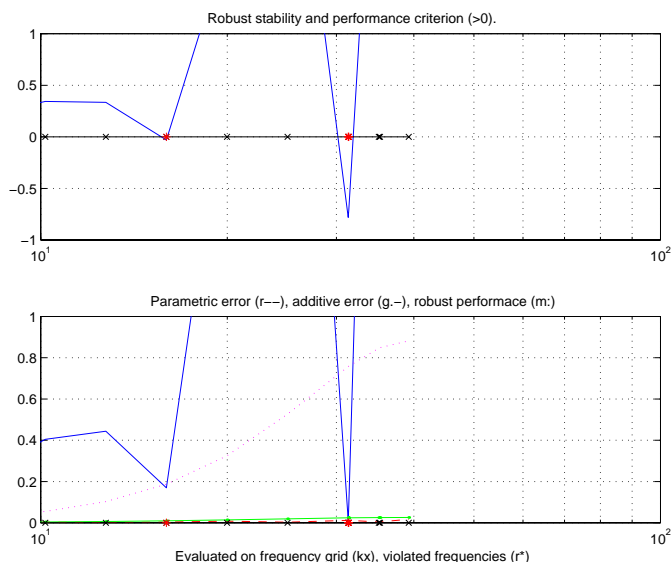

Figure 8: Analysis of first order controller (zoomed version of Fig. 7). Upper plots: Criterion function for robust stability (rhs of (19)), evaluated at a set of test frequencies (crosses). Lower plots: Single contributions in (8): $\mathcal{R}_{m}^{T} \theta$ (solid), Parametric error $\left\|\mathcal{R}_{s} \theta\right\|_{2}$ (dashed), additive error $\left\|\mathcal{R}_{u s} \theta\right\|_{2}$ (dash-dotted), robust performance $\left\|\mathcal{R}_{P} \theta\right\|_{2}$ (dotted)

\section{References}

[1] J. Ackermann. Robust Control. Systems with Uncertain Physical Parameters. Springer Verlag, Berlin, Germany, 1996.

[2] G. Bianchini, P. Falugi, A. Tesi, and A. Vicino. Restricted complexity robust controllers for uncertain plants with rank one real perturbations. In Proc. of the 37th IEEE Conference on Decision and Control, pages 1213-1218, Tampa, FL, USA, Dec. 1998.

[3] X. Bombois, M. Gevers, G. Scorletti, and B. D. O. Anderson. Controller validation for stability and performance based on an uncertainty region designed from an identified model. In Proc. of the System Identification Symposium SYSID, Santa Barbara, CA, USA, June 2000.

[4] H. Chapellat, M. A. Dahleh, and S. P. Bhattacharyya. Robust stability under structured and unstructured perturbations. IEEE Trans. on Automatic Control, 35(10):1100-1108, Oct. 1990.

[5] A. Garulli and W. Reinelt. On model error modeling in set membership identification. In Proc. of the System Identification Symposium SYSID, pages WeMD1-3, Santa Barbara, CA, USA, June 2000.

[6] A. Garulli, A. Tesi, and A. Vicino, editors. Robustness in Identification and Control. Number 245 in Lecture Notes in Control and Information Sciences. Springer-Verlag, 1999.

[7] Z. Geng and L. Huang. Robust stability of the systems with mixed uncertainties under the IQC descriptions. Int. J. of Control, 73(9):776786, Sept. 2001.

[8] A. Ghulchak and A. Rantzer. Robust controller design via linear programming. In Proc. of the 38th IEEE Conference of Decision and Control, Phoenix, AZ, USA, Dec. 1999.

[9] M. Green and D. J. N. Limebeer. Linear Robust Control. Prentice Hall, Englewood Cliffs, NJ, USA, 1995.

[10] D. Henrion and O. Bachelier. Low-order robust controller design for interval plants. Int. J. of Control, 74(1):1-9, Jan. 2001.

[11] L. Ljung. Model validation and model error modeling. In B. Wittenmark and A. Rantzer, editors, Proc. of the Aström Symposium on Control, pages 15-42, Lund, Sweden, Aug. 1999. Studentliteratur, Lund, Sweden.

[12] L. Ljung. System Identification - Theory For the User. Prentice Hall, Upper Saddle River, NJ, USA, 2nd edition, 1999.

[13] M. S. Lobo, L. Vandenberghe, S. Boyd, and H. Lebret. Applications of second-order cone programming. Linear Algebra and its Applications, 284:193-228, Nov. 1998.

[14] S. Malan, M. Milanese, and M. Taragna. Robust analysis and design of control systems using interval arithmetic. Automatica, 33(7):13631372, 1997.

[15] M. Milanese, J. P. Norton, H. Piet-Lahanier, and E. Walter, editors. Bounding Approaches to System Identification. Plenum Press, New York, NY, USA, 1996.

[16] A. Rantzer and A. Megretski. A convex parameterization of robustly stabilizing controllers. IEEE Trans. on Automatic Control, 39(9):18021808, Sept. 1994.

[17] L. Wang and G. C. Goodwin. Integrating identification with robust control: a mixed $\mathrm{H}_{2} / \mathrm{H}_{\infty}$ approach. In Proc. of the IEEE Conference on Decision and Control, Sydney, Australia, Dec. 2000. 